\title{
Astronomy, Pseudoscience, and Rational Thinking
}

\author{
Jayant V. Narlikar \\ Inter-University Centre for Astronomy and Astrophysics, India \\ E-mail: jvn@iucaa.ernet.in
}

In 1944, three years before India became independent of the British rule, Jawaharlal Nehru wrote in his now famous book Discovery of India: "The impact of science and the modern world have brought a greater appreciation of facts, a more critical faculty, a weighing of evidence, a refusal to accept tradition merely because it is tradition". But even today it is strange how we suddenly become overwhelmed by tradition, and the critical faculties of even intelligent people cease to function. He then went on to express the hope that "Only when we are politically and economically free will the mind function normally and critically".

India is now well into the sixth decade of the post-independence era, and where do we stand vis-a-vis rational thinking? Here are some pointers from the present times:

- Many marriages, even amongst the educated graduates are decided after the matching of horoscopes.

- Astrologers are consulted for deciding an auspicious date for launching a new state, for swearing in a new cabinet, for purchasing a house or a car, for undertaking travel.

- An entire village goes into a state of panic and people abandon their homes and run away under the impression that an alignment of planets on one side is going to cause a major catastrophe.

- Houses are built or remodeled to satisfy the dictates of "Vastu-shastra" or the (pseudo-)science of architecture, which in fact is a collection of unproven claims linking the ups and downs in the lives of the inhabitants of a house to where specific rooms in the house happen to be.

- The "miracle" of the idol of the elephant-god Ganesha drinking milk through his trunk can send large crowds to temples, or of a "holy godman" producing watches out of nothing for his devotees is taken seriously even by intellectuals.

- The apex body of higher education, the University Grants Commission declares astrology as a science and offers funds for instituting its teaching in universities.

The disturbing fact about these symptoms is that the trend towards superstitions and beliefs in pseudo-sciences is growing and the younger generation is getting more and more attracted to these ideas. How to counteract this trend? 
I believe education in astronomy right from the school level can play a major role in this enterprise. An indication of how education in science can help distinguish it from pseudo-science can be seen from the following example of chemistry.

In India non-governmental organizations devoted to eradication of superstitions, have two visiting teams doing the rounds of various schools. The first team performs "miracles" which the second team following it, explains by chemistry experiments. When the unexpected or the unusual gets demonstrated as a known fact of science, the belief in miracles crumbles. Such direct demonstrations have proven to be very useful and effective. [The Ganesha phenomenon was likewise demonstrated and clarified by scientific experiments.]

Astronomy provides similar instances that distinguish it as a science from pseudo-science. These instances come through several channels.

- The solar eclipse is known to generate awe and even fear in the human mind, provided it remains unexplained. Its explanation as a shadow effect and the demonstration of its complete and accurate predictability leads to a new mind set of the viewer who is then able to enjoy this extraordinary phenomenon. Several superstitions related to this event can be effectively eliminated by encouraging information about the event and active participation of school children in it as viewers. They may be encouraged to enlighten their parents and grandparents too!

- Just as the solar and lunar eclipses are completely predictable, so are transits of Venus and Mercury. The fact that laws of physics apply to such events will help the children appreciate them as real physical experiments.

- The same point can be driven home further by illustrating with the examples of planetary fly-bys by man-made spacecraft. Here for example, one can demonstrate that the planets and the spacecraft both obey the same laws of dynamics. The power of mathematics in calculating the precise rendezvous will also not be lost on the student.

- The seaside towns regularly have tides. One can use the timings of these tides and the positions of the Sun and the Moon to demonstrate their origin in terms of Newton's law of gravitation.

In the Indian context, special efforts are needed to counteract the growing influence of astrology. To contrast a pseudo-science like astrology with a hard science like astronomy, the above examples from astronomy may be put side by side with how astrology operates. Thus children could be asked to apply the following criteria to astrology:

- Scientific predictions are falsifiable and are therefore worded in a precise manner. Are astrological predictions so worded or are they vague and could be made consistent with any result?

- Even if they are tested for success or failure, how often are they successful and how often not? The example of coin-tossing may illustrate the circumstance that a prediction may turn out correct purely by chance. 
- Is the making of a prediction logical and based on some precisely stated assumptions?

- Do the successes / failures of a prediction depend on who makes them? That is, is there objectivity about them?

- Field tests on large samples and their overall success-evaluation have established the validity of empirical relationships in physics, which were subsequently explained by basic laws. What has been astrology's record with such field tests?

Ample evidence exist in literature to show why astrology is not a science. It will be worthwhile spending some part of astronomy education in demonstrating to children why this is so.

Science teaches rational thinking and the application of the scientific outlook goes well beyond the laboratory walls. Astronomy boldly applies the laws of science to the grand laboratory that is our universe. Some natural phenomena can be awe-inspiring and if not explained scientifically, can lead to superstitions and irrational thinking. Children could learn how to think rationally and not get carried away by superstitions, pseudo-science and loose thinking, if they are given some introduction to astronomy as a science and are encouraged to apply the scientific criteria to see why astrology is not a science. 